\title{
Comparison of the Mutation Profiles of Triple- Negative Breast Cancers and Hormone Receptor- Positive/Human Epidermal Growth Factor Receptor 2-Negative Breast Cancers at the T2N0-1M0 Stage
}

\section{Seungju Lee}

Pusan National University Yangsan Hospital

Hyun Yul Kim

Pusan National University Yangsan Hospital

Youn Joo Jung

Pusan National University Yangsan Hospital

Hyun-June Paik

Pusan National University Yangsan Hospital

Chang Shin Jung

Pusan National University Yangsan Hospital

Jee Yeon Kim

Pusan National University Yangsan Hospital

\section{Sun Min Lee}

Pusan National University Yangsan Hospital

Seung Hwan Oh ( $\sim$ paracelsus@pusan.ac.kr)

Pusan National University Yangsan Hospital

\section{Research Article}

Keywords: Triple-negative breast cancer, TP53, PIK3CA

Posted Date: December 28th, 2020

DOI: https://doi.org/10.21203/rs.3.rs-129609/v1

License: (c) (i) This work is licensed under a Creative Commons Attribution 4.0 International License. Read Full License 


\section{Abstract}

\section{Background}

Triple-negative breast cancer (TNBC) has higher loco-regional recurrence and visceral metastasis compared to other breast cancer subtypes; however, little is known about the molecular pathogenesis and therapeutic targets of TNBC. Therefore, we compared the mutation profiles of early TNBC with those of hormone receptor-positive (HR+)/human epidermal growth factor receptor 2-negative (HER2ロ) breast cancer using a customized next-generation sequencing capture panel.

Methods

DNA was obtained from the primary tumor tissues of 34 patients diagnosed with pT2N0-1M0 HR+/HER2 breast cancer or TNBC. To enrich the 48 breast cancer-associated genes, 21,192 probes were designed using the SureSelect design tool. After library preparation using the SureSelect XT kit (Agilent), paired-end DNA sequencing was performed on a HiSeq platform (Illumina). The mean depth of the target regions was $1,766(x)$. The subsequent output containing genetic variation was analyzed using a pipeline of bioinformatics tools. Significant mutations with allele frequencies of more than $30 \%$ were checked for their germline counterparts in the peripheral blood. Circulating cell-free nucleic acids were extracted and analyzed with a therascreen ${ }^{\circledR}$ PIK3CA RGQ PCR kit (QIAGEN).

Results

Significant mutations were found in TP53, PIK3CA, AR, BRCA1, PTEN, BRCA2, BRIP2, KIT, MET, AKT1, ALK, BARD1, BRAF, CD274, ERBB2, FGFR1, IDH2, NOTCH1, RET, and STK11 (in descending order of occurrence). TP53 mutations were identified in the TNBC group more frequently than in the HR+/HER2 group $(p=0.003)$. The presence of TP53 mutations was associated with a higher tumor grade $(p=0.008)$, p53 positivity $(p<0.0001)$, and a higher $(\geq 15) \mathrm{Ki}-67$ index $(p=0.004)$. PIK3CA was the most frequently mutated gene in HR+/HER2 $\$ breast cancer $(8 / 22,36.4 \%)$, but not in TNBC $(1 / 12,8.3 \%)$. However, circulating cell-free PIK3CA mutations were not detected in either group.

Conclusions

The TP53 mutation is associated with higher tumor grade and $\mathrm{Ki}-67$ expression in both groups, and with larger tumor size in TNBC, but not in HR+/HER2- breast cancer. In the foundation of TP53 mutation, concomitant mutation numbers are proportional to tumor size, reflecting clonal progression. Breast cancer-associated mutations such as those in TP53 and PIK3CA have different biological implications for the proliferation and clonal diversification of these two distinct groups of breast cancer.

\section{Background}

Triple-negative breast cancer (TNBC) has greater loco-regional recurrence and more distant metastasis compared to the other breast cancer subtypes [1-3]. Furthermore, TNBC lacks a targeted therapy because 
of the absence of the hormone receptor (HR) and human epidermal growth factor receptor 2 (HER2). Therefore, many researchers have tried to classify breast cancers according to molecular genetic events and sought to identify novel drug combinations by analyzing the accompanying gene mutations [4-7].

With the advent of next-generation sequencing (NGS), numerous breast cancer-associated mutations have been discovered. The most commonly mutated genes in primary breast cancers are tumor protein p53 (TP53) and phosphatidylinositol-4,5-bisphosphate 3-kinase catalytic subunit alpha (PIK3CA) [8]. Approximately $40 \%$ of HR-positive breast cancers harbor PIK3CA mutations. Recently, the PI3K inhibitor alpelisib with fulvestrant received Food and Drug Administration (FDA) approval for PIK3CA-mutated HRpositive (+)/HER2-negative (-) advanced breast cancer after prior endocrine therapy [9]. The FDA also approved companion diagnostic tests for PIK3CA mutations for specimens of either tumor tissue or plasma cell-free nucleic acids [10].

Therefore, we analyzed breast cancer-associated gene mutations in tissues of diagnosed TNBC and HR+/HER2- breast cancer using a customized NGS panel and ran the therascreen ${ }^{\circledR}$ PIK3CA RGQ PCR assay (QIAGEN) with cell-free nucleic acids extracted from PIK3CA-mutated patients. Finally, the clinicopathological parameters and accompanying gene mutations of relatively early stage TNBC and HR+/HER2- breast cancer were compared.

\section{Methods}

\section{Patients}

This study included 34 Korean patients who were diagnosed with pT2N0-1M0 HR+/HER2- or triplenegative primary invasive ductal cancer at Pusan National University Yangsan Hospital, Korea, in 2016. They were treated with surgery and adjuvant chemotherapy, radiation therapy, or endocrine therapy. We assessed the histology, tumor grade, tumor size, lymph node status, type of surgery, and status of chemotherapy, radiation therapy, and endocrine therapy. We used Allred scoring to grade the hormone receptor status, with scores of 3 to 8 considered positive. HER2 was analyzed immunohistochemically, and the results were scored on a scale of $0,1+, 2+$, and $3+$. When the result was $2+$ and considered equivocal, silver in situ hybridization was performed. TNBC included tumors that were negative for hormone receptors and HER2. This study was approved by the Institutional Review Board of Pusan National University Yangsan Hospital.

\section{Tumor DNA extraction and customized targeted sequencing}

DNA was extracted from frozen primary tumor tissues. Using the SureSelect design tool, 21,192 probes were designed to enrich 48 breast cancer-associated genes: AKT1, ALK, AR, BARD1, BRAF, BRCA1, BRCA2, BRIP1, CCND1, CD274, CDK4, CDK6, CDKN2A, DDR2, EGFR, ERBB2, ERBB3, ERBB4, ESR1, FGFR1, FGFR2, FGFR3, GNA11, GNAQ, HRAS, IDH1, IDH2, KIT, KRAS, MAP2K1, MET, MTOR, MYC, NOTCH1, NRAS, PALB2, PDGFRA, PIK3CA, PTEN, RAD51C, RAD51D, RAF1, RET, ROS1, SMO, STK11, TP53, and TSC1. A DNA library was prepared using the SureSelect XT kit (Agilent Technologies Inc., Santa Clara, CA, USA), and paired- 
end DNA sequencing was performed on the HiSeq platform (Illumina, San Diego, CA, USA). The mean depth of the target regions was $1,766(x)$. The subsequent output containing genetic variants was analyzed using a pipeline of bioinformatics tools, including read mapping, variant calling, and annotation.

Circulating cell-free nucleic acid extraction from plasma and PIK3CA testing using the therascreen ${ }^{\circledR}$ PIK3CA RGQ PCR assay

Circulating cell-free nucleic acids were extracted with a QIAamp DSP Circulating Nucleic Acids kit (QIAGEN, Hilden, Germany) after double centrifugation from the plasma of 24 breast cancer patients including seven with PIK3CA mutations discovered by targeted sequencing. The quality of the extracted cell-free nucleic acids was checked using the 4150 TapeStation System (Agilent Technologies Inc.). The therascreen ${ }^{\circledR}$ PIK3CA RGQ PCR assay using real-time PCR (QIAGEN) was performed to detect C420R, E542K, E545A/D/G/K, Q546E/R, and H1047L/R/Y according to the manufacturer's instructions.

\section{Sanger sequencing for determining germline/somatic mutations}

All TP53 and other pathogenic and likely pathogenic mutations with variant allele frequencies above $30 \%$ were checked for the presence of their germline counterparts by Sanger sequencing of available peripheral blood. We directly sequenced the genomic DNA using a BigDye ${ }^{\mathrm{TM}}$ Terminator v3.1 Cycle Sequencing kit (Applied Biosystems, Foster City, CA, USA) and an ABI 3700 Genetic Analyzer (Applied Biosystems).

\section{Statistical analysis}

The tumor characteristics and gene mutations were compared using chi-square tests and Pearson correlations, and a $p$-value less than 0.05 was considered to denote significance. The data were analyzed using MedCalc (ver. 12.7.0.0, MedCalc Software, Ostend, Belgium).

\section{Results}

\section{Patient characteristics}

Table 1 shows the clinical and pathological characteristics of the enrolled breast cancer patients. All patients were diagnosed with invasive ductal cancer after surgery in 2016. Fourteen patients were diagnosed with pT2N0 and 20 with pT2N1. The median patient age was 50 (range 38-67) and 58 (range 37-75) years in the TNBC and HR+/HER2- groups, respectively. Although patient age did not differ significantly between groups, the age range in the TNBC group indicates more radiotherapy followed by breast-conserving surgery. Regarding the tumor grade, the majority of the TNBC patients had grade 3 tumors (11 patients, 91.7\%), whereas 10 (45.5\%) HR+/HER2- breast cancer patients had grade 3 tumors $(p=0.029)$. TNBC was closely related to $p 53$ positivity and a high Ki-67 index (Table 1, Fig. 1$)$. Conversely, lymphovascular invasion was more frequent in HR+/HER2- breast cancer. Loco-regional recurrence 
occurred in two patientsøne in each group. One patient with TNBC had lung and bone metastases during follow-up. Regarding primary tumor tissue mutations, nine patients had PIK3CA mutations and 14 had TP53 mutations. As expected from the immunohistochemistry, TNBC was associated with TP53 mutations detected by targeted sequencing (Table 1). 
Table 1

Demographic and clinicopathological characteristics of the two groups of breast cancer patients

\begin{tabular}{|c|c|c|c|}
\hline Characteristics & HR+/HER2- & TNBC & $p$-value \\
\hline Age at diagnosis (average) & 58 yrs & 50 yrs & 0.286 \\
\hline Tumor size $(\mathrm{cm})$ & 2.8 & 3.0 & 0.163 \\
\hline $\mathrm{N}$ stage & & & 0.966 \\
\hline NO & 9 & 5 & \\
\hline N1 & 13 & 7 & \\
\hline No. of involved lymph nodes & & & 0.594 \\
\hline 0 & 9 & 5 & \\
\hline 1 & 9 & 3 & \\
\hline 2 & 2 & 3 & \\
\hline 3 & 2 & 1 & \\
\hline Grade & & & $0.029 *$ \\
\hline 1 & 1 & 0 & \\
\hline 2 & 11 & 1 & \\
\hline 3 & 10 & 11 & \\
\hline p53 & & & $0.002^{*}$ \\
\hline negative & 16 & 2 & \\
\hline positive & 6 & 10 & \\
\hline Ki-67 & & & $0.001^{*}$ \\
\hline$<15$ & 12 & 0 & \\
\hline$\geq 15$ & 10 & 12 & \\
\hline Lymphovascular invasion & & & 0.017 \\
\hline no & 9 & 10 & \\
\hline yes & 13 & 2 & \\
\hline Multiplicity & & & 0.180 \\
\hline single & 19 & 12 & \\
\hline$* p<0.05$ & & & \\
\hline
\end{tabular}




\begin{tabular}{|llll|}
\hline Characteristics & HR+/HER2- & TNBC & $p$-value \\
\hline multiple & 3 & 0 & \\
\hline Surgery & & & $0.004^{*}$ \\
\hline breast-conserving surgery & 9 & 11 & \\
\hline mastectomy & 13 & 1 & \\
\hline Radiotherapy & & & $0.005^{\star}$ \\
\hline yes & 12 & 12 & \\
\hline no & 10 & 0 & \\
\hline Adjuvant chemotherapy & & & 0.180 \\
\hline yes & 19 & 12 & \\
\hline no & 3 & 0 & \\
\hline TP53 mutation & & & $0.003^{*}$ \\
\hline negative & 17 & 3 & \\
\hline positive & 5 & 9 & \\
\hline PIK3CA mutation & & 11 & \\
\hline negative & 14 & & \\
\hline positive & 8 & & \\
\hline * $p<0.05$ & & & \\
\hline
\end{tabular}

Comparison of the mutation profiles of primary breast cancer tissues between HR+/HER2- and TNBC (supplementary Table S1)

Significant [pathogenic, likely pathogenic, and variation of uncertain significance (VUS)] mutations were found in TP53, PIK3CA, AR, BRCA1, PTEN, BRCA2, BRIP2, KIT, MET, AKT1, ALK, BARD1, BRAF, CD274, ERBB2, FGFR1, IDH2, NOTCH1, RET, and STK11 (Fig. 2). No significant variation in the estrogen receptor alpha (ESR1) gene was found in either group. Of the 34 breast cancer patients, 79\% (27/34) harbored at least one pathogenic or likely pathogenic mutation.

PIK3CA mutations were detected in eight (36.4\%) of $22 \mathrm{HR}+/ \mathrm{HER} 2-$ breast cancers and one (8.3\%) of 12 TNBC. One HR+/HER2- patient had double PIK3CA mutations (c.1357G >A and c.3140A > G). TP53 mutations were detected in five (22.7\%) HR+/HER2- breast cancers and nine (75.0\%) TNBC. One TNBC patient had a TP53 missense mutation $(c .641 A>G)$ and a PIK3CA frame-shift mutation (c.3627dupA). All TP53 mutations were confirmed as somatic mutations via direct sequencing of peripheral blood, regardless of their variant allele frequencies (range 14.41-86.58\%). All three BRCA2 mutations were 
germline mutations, co-existed with somatic PIK3CA, TP53, and ERBB2 mutations, and were found only in the HR+/HER2 - group. However, only two of the four BRCA1 mutations were germline mutations; one was a frame-shift mutation (c.3627dupA) that was also changed to a homozygous mutation in tumor tissue by somatic loss of heterozygosity ( $(\mathrm{OH})$.

We analyzed the relationship between the TP53 and PIK3CA mutations based on tumor DNA and the patients' pathological parameters (Table 2). The presence of TP53 mutations was associated with a higher tumor grade $(p=0.008)$, p53 positivity $(p<0.001)$, and a higher Ki-67 index $(p=0.004)$. The presence of PIK3CA mutations was associated with lymphovascular invasion $(p=0.018)$ and a lower Ki67 index $(p=0.022)$.

Table 2

Pathological parameters according to mutation types.

\begin{tabular}{|c|c|c|c|c|c|c|}
\hline \multirow[t]{2}{*}{ Pathology } & \multicolumn{2}{|c|}{ TP53 mutation } & \multirow[t]{2}{*}{$p$-value } & \multicolumn{2}{|c|}{ PIKЗCA mutation } & \multirow[t]{2}{*}{$p$-value } \\
\hline & + & - & & + & - & \\
\hline Grade & & & $0.008^{*}$ & & & 0.055 \\
\hline 1 & 0 & 1 & & 1 & 0 & \\
\hline 2 & 1 & 11 & & 5 & 7 & \\
\hline 3 & 13 & 8 & & 3 & 18 & \\
\hline Lymphovascular invasion & & & $0.026^{*}$ & & & $0.018^{*}$ \\
\hline no & 11 & 8 & & 2 & 17 & \\
\hline yes & 3 & 12 & & 7 & 8 & \\
\hline Tissue p53 & & & $<0.001^{*}$ & & & 0.082 \\
\hline negative & 2 & 16 & & 7 & 11 & \\
\hline positive & 12 & 4 & & 2 & 14 & \\
\hline Tissue Ki-67 & & & $0.004^{*}$ & & & $0.022^{*}$ \\
\hline$<15$ & 1 & 11 & & 6 & 6 & \\
\hline$\geq 15$ & 13 & 9 & & 3 & 19 & \\
\hline$* p<0.05$ & & & & & & \\
\hline
\end{tabular}

\section{Cell-free nucleic acid PIK3CA mutations from breast cancer patient plasma}

Cell-free nucleic acid PIK3CA mutations were not detected in the 24 plasma samples from patients with breast cancers at these stages, although seven harbored PIK3CA mutations detected by targeted 
sequencing.

\section{Comparison of tumor size according to TP53 and accompanying mutations}

Although every subject was classified as the same T2 stage (widest primary tumor size 2-5 cm), TP53mutated tumors were smaller than TP53-non-mutated ones in the HR+/HER2- group; however, TP53mutated tumors were relatively large in the TNBC group (Fig. 3A). In other words, the TP53-mutated TNBC tumors were larger than the TP53-mutated HR+/HER2- breast cancers $(p=0.0493)$ (Fig. 3A). Tumor size was correlated with the total number of significant mutations including TP53 and other accompanying mutations in TP53-mutated breast cancers ( $r=0.7156, p=0.004)$ (Fig. 3B).

\section{Discussion}

TNBC is clinically aggressive with a shorter interval to recurrence and metastasis compared to HR + breast cancer $[1-3,11]$. TNBC has no specific targeted therapy other than surgery, chemotherapy, and radiotherapy because it does not express HR or HER2. However, high-throughput molecular diagnostic technologies such as NGS and microarray analyses have expanded our knowledge of the genetic alterations in cancers and provided new insight into the molecular pathogenesis of cancers $[8,12,13]$. Using NGS, the most commonly mutated genes in primary breast cancer are TP53 and PIK3CA [8]. The frequency of mutations in these genes depends on the breast cancer subtype. TNBC has a higher predominance of TP53 mutations compared to luminal A breast cancer [5, 8, 14-16]. Similarly, we also found a higher incidence of TP53 mutations in TNBC (75.0\%) compared to HR+/HER2- breast cancer $(22.7 \%)$ in sporadic breast cancers in ethnic Koreans. This was similar to previous findings that TNBC is associated with a higher incidence of TP53 mutations (80\%) than are luminal A breast cancers (12\%) [8].

Regarding the clinicopathological implications of these two main breast cancer-associated genes, TP53 mutations in primary tumor tissues were associated with p53 positivity $(p<0.001)$ and a higher Ki-67 index $(p=0.004)$, as expected. TP53-mutated breast cancers also had a higher tumor grade $(p=0.008)$, whereas PIK3CA-mutated breast cancers exhibited more lymphovascular invasion $(p=0.008)$.

All TP53 and PIK3CA mutations were somatic mutations and were categorized as pathogenic or likely pathogenic variants. In addition, $A K T 1, A L K, B R C A 1, E R B B 2$, and PTEN were somatically mutated genes and existed without additional pathogenic or likely pathogenic mutations. AR, KIT, and STK11 were also somatically mutated genes, with co-existing pathogenic mutations. The other mutations were only VUS or germline mutations. All three BRCA2 mutations were germline mutations, accompanied by additional pathogenic or likely pathogenic somatic mutations, and were found only in HR+/HER2- breast cancers. Four BRCA1 mutations were found in both groups, one showed LOH of a germline mutation in TNBC and another was a somatic mutation in HR+/HER2- breast cancer. Although BRCA1/2 mutations are strongly associated with TNBC and a high proportion of mutation carriers can develop TNBC, germline BRCA1/2 mutations account for only $15.4 \%$ of TNBC $[17,18]$. In this study, only two $(16.7 \%)$ BRCA1 mutations were found in sporadic TNBC, and one of them was a germline mutation with $\mathrm{LOH}$. 
Both the TNBC and HR+/HER2- groups had no significant variants of the ESR1 gene, which are generally found in advanced breast cancer patients treated with endocrine therapy [19]. We monitored patients for a median of 43.5 (range 10-50) months. The follow-up period was short, although recurrences or metastases of TNBC are known to occur within 2-3 years. We had a limited ability to identify the relationship between gene mutations and prognosis because the study subjects had relatively early breast cancer diagnosed as pT2N0-1M0. Recent studies have revealed the relationship between gene mutations and prognosis through analyses of circulating tumor DNA (ctDNA) in advanced breast cancers [20-22] Therefore, we analyzed circulating cell-free nucleic acids in preserved blood plasma with the therascreen ${ }^{\circledR}$ PIK3CA RGQ PCR kit (QIAGEN). Circulating cell-free PIK3CA mutations were absent in either groups, which might have been due to the relatively early stage of disease because this diagnostic assay is approved only for advanced disease. We think that the usefulness of therascreen $\circledast$ PIK3CA mutation detection with cell-free nucleic acids at the early stage is still questionable.

Somatic mutations of TP53, PIK3CA, and PTEN are considered founder mutations in primary breast cancers and additional mutations occur in genes involved in other pathways, such as those controlling cell shape and the cytoskeleton [23]. In TNBC, TP53 mutations and methylation of the BRCA1 promoter region are early oncogenic events, and additional structural and epigenetic events occur during clonal progression, resulting in the amplification of oncogenes such as $M Y C$, NOTCH2, and NOTCH3, the hampering of tumor suppressor genes, including RB1, PTEN, and KMT2C, and the enhancement of the TGFA oncogene [24]. In this study, the TP53 mutation was predominant in TNBC, and the tumor size seemed to be proportional to the total number of significant mutations in TP53-mutated breast cancer, although the cancers were at the same tumor stage $(T 2,2-5 \mathrm{~cm})$. Therefore, we inferred that tumor size reflects clonal progression and diversification at earlier tumor stages in TP53-mutated breast cancers.

\section{Conclusion}

The TP53 mutation is more frequent in TNBC than in HR+/HER2- cancer. It is associated with a higher tumor grade and greater $\mathrm{Ki}-67$ expression in both groups, and with a larger tumor size in TNBC, but not in $\mathrm{HR}+/ \mathrm{HER} 2$ - breast cancer. With TP53 mutation, concomitant mutation numbers are proportional to tumor size, reflecting clonal progression. Breast cancer-associated mutations such as those in TP53 and PIK3CA have different biological implications for the proliferation and clonal diversification of these two distinct groups of breast cancer.

\section{Abbreviations}

TNBC: Triple-negative breast cancer

HR+/HER2-: hormone receptor-positive/human epidermal growth factor receptor 2-negative

NGS: next-generation sequencing

TP53: tumor protein p53 
PIK3CA: phosphatidylinositol-4,5-bisphosphate 3-kinase catalytic subunit alpha

FDA: Food and Drug Administration

VUS: variation of uncertain significance

LOH: loss of heterozygosity

\section{Declarations}

\section{Availability of data and materials}

The authors confirm that all data supporting the findings of this study are available within the article and its supplementary materials.

\section{Ethics declarations}

\section{Ethics approval and consent to participate}

All tested patients provided a signed informed consent form prior to gene mutation tests and permission for the anonymous use of their data for research purpose. This study was reviewed and approved by the Institutional Review Board of Pusan National University Yangsan Hospital (04-2020-003). Bio-specimens and clinical data were obtained from the Institutional Biobank Project (OF-2020-01) in accordance with the approved research protocol. All work in this study has been performed in accordance with the Declaration of Helsinki.

\section{Consent for publication}

Not applicable.

\section{Competing interests}

The authors declare that there are no conflicts of interest.

\section{Funding}

This study was supported by the Research Institute for the Convergence of Biomedical Science and Technology (30-2020-014), Pusan National University Yangsan Hospital. 


\section{Authors' contributions}

S Lee and SH Oh drafted the manuscript. SH Oh performed analysis and interpretation of the variant data and prepared all supporting figures. S Lee and SM Lee designed the study, and analyzed the clinicopathological data of patients. HY Kim, YJ Jung, HJ Paik, and CS Jung provided the patient materials and treatment. JY Kim interpreted all the pathologic findings. All authors have read and approved the final manuscript.

\section{Acknowledgments}

The authors thank Yelin Ryu, MT in the Department of Laboratory Medicine, Pusan National University Yangsan Hospital for her technical assistance.

\section{Supplementary Information}

Additional file 1: Table S1.docx

Significant sequence variations detected in this study.

\section{References}

1. Ihemelandu CU, Naab TJ, Mezghebe HM, Makambi KH, Siram SM, Leffall LD, Jr., DeWitty RL, Jr., Frederick WA: Treatment and survival outcome for molecular breast cancer subtypes in black women. Ann Surg 2008, 247(3):463-469. https://doi.org/10.1097/SLA.0b013e31815d744a

2. Lowery AJ, Kell MR, Glynn RW, Kerin MJ, Sweeney KJ: Locoregional recurrence after breast cancer surgery: a systematic review by receptor phenotype. Breast Cancer Res Treat 2012, 133(3):831-841. https://doi.org/10.1007/s10549-011-1891-6

3. McGuire A, Lowery AJ, Kell MR, Kerin MJ, Sweeney KJ: Locoregional Recurrence Following Breast Cancer Surgery in the Trastuzumab Era: A Systematic Review by Subtype. Ann Surg Oncol 2017, 24(11):3124-3132. https://doi.org/10.1245/s10434-017-6021-1

4. Le Du F, Eckhardt BL, Lim B, Litton JK, Moulder S, Meric-Bernstam F, Gonzalez-Angulo AM, Ueno NT: Is the future of personalized therapy in triple-negative breast cancer based on molecular subtype? Oncotarget 2015, 6(15):12890-12908. https://doi.org/10.18632/oncotarget.3849

5. Marotti JD, de Abreu FB, Wells WA, Tsongalis GJ: Triple-Negative Breast Cancer: Next-Generation Sequencing for Target Identification. Am J Pathol 2017, 187(10):2133-2138. https://doi.org/10.1016/j.ajpath.2017.05.018

6. Quintero M, Adamoski D, Reis LMD, Ascencao CFR, Oliveira KRS, Goncalves KA, Dias MM, Carazzolle MF, Dias SMG: Guanylate-binding protein-1 is a potential new therapeutic target for triple-negative breast cancer. BMC Cancer 2017, 17(1):727. https://doi.org/10.1186/s12885-017-3726-2 
7. Weisman PS, Ng CK, Brogi E, Eisenberg RE, Won HH, Piscuoglio S, De Filippo MR, loris R, Akram M, Norton $\mathrm{L}$ et al: Genetic alterations of triple negative breast cancer by targeted next-generation sequencing and correlation with tumor morphology. Mod Pathol 2016, 29(5):476-488. https://doi.org/10.1038/modpathol.2016.39

8. Cancer Genome Atlas N: Comprehensive molecular portraits of human breast tumours. Nature 2012, 490(7418):61-70. https://doi.org/10.1038/nature11412

9. Copur MS: Alpelisib to treat breast cancer. Drugs Today (Barc) 2020, 56(6):357-363. https://doi.org/10.1358/dot.2020.56.6.3137526

10. Martinez-Saez O, Chic N, Pascual T, Adamo B, Vidal M, Gonzalez-Farre B, Sanfeliu E, Schettini F, Conte B, Braso-Maristany $\mathrm{F}$ et al: Frequency and spectrum of PIK3CA somatic mutations in breast cancer. Breast Cancer Res 2020, 22(1):45. https://doi.org/10.1186/s13058-020-01284-9

11. Elias AD: Triple-negative breast cancer: a short review. Am J Clin Oncol 2010, 33(6):637-645. https://doi.org/10.1097/COC.0b013e3181b8afcf

12. de Abreu FB, Peterson JD, Amos $\mathrm{Cl}$, Wells WA, Tsongalis GJ: Effective quality management practices in routine clinical next-generation sequencing. Clin Chem Lab Med 2016, 54(5):761-771. https://doi.org/10.1515/cclm-2015-1190

13. Klijn C, Durinck S, Stawiski EW, Haverty PM, Jiang Z, Liu H, Degenhardt J, Mayba O, Gnad F, Liu J et al: A comprehensive transcriptional portrait of human cancer cell lines. Nat Biotechnol 2015, 33(3):306-312. https://doi.org/10.1038/nbt.3080

14. Carrick DM, Mehaffey MG, Sachs MC, Altekruse S, Camalier C, Chuaqui R, Cozen W, Das B, Hernandez BY, Lih CJ et al: Robustness of Next Generation Sequencing on Older Formalin-Fixed Paraffin-Embedded Tissue. PLoS One 2015, 10(7):e0127353. https://doi.org/10.1371/journal.pone.0127353

15. Ellsworth DL, Turner CE, Ellsworth RE: A Review of the Hereditary Component of Triple Negative Breast Cancer: High- and Moderate-Penetrance Breast Cancer Genes, Low-Penetrance Loci, and the Role of Nontraditional Genetic Elements. J Oncol 2019, 2019:4382606. https://doi.org/10.1155/2019/4382606

16. Shang M, Chang C, Pei Y, Guan Y, Chang J, Li H: Potential Management of Circulating Tumor DNA as a Biomarker in Triple-Negative Breast Cancer. J Cancer 2018, 9(24):4627-4634. https://doi.org/10.7150/jca.28458

17. Greenup R, Buchanan A, Lorizio W, Rhoads K, Chan S, Leedom T, King R, McLennan J, Crawford B, Kelly Marcom $P$ et al: Prevalence of BRCA mutations among women with triple-negative breast cancer (TNBC) in a genetic counseling cohort. Ann Surg Oncol2013, 20(10):3254-3258. https://doi.org/10.1245/s10434-013-3205-1

18. Newman LA, Reis-Filho JS, Morrow M, Carey LA, King TA: The 2014 Society of Surgical Oncology Susan G. Komen for the Cure Symposium: triple-negative breast cancer. Ann Surg Oncol 2015, 22(3):874-882. https://doi.org/10.1245/s10434-014-4279-0 
19. Fribbens C, Garcia Murillas I, Beaney M, Hrebien S, O'Leary B, Kilburn L, Howarth K, Epstein M, Green $\mathrm{E}$, Rosenfeld $\mathrm{N}$ et al: Tracking evolution of aromatase inhibitor resistance with circulating tumour DNA analysis in metastatic breast cancer. Ann Oncol 2018, 29(1):145-153. https://doi.org/10.1093/annonc/mdx483

20. Abbosh C, Swanton C, Birkbak NJ: Circulating tumour DNA analyses reveal novel resistance mechanisms to CDK inhibition in metastatic breast cancer. Ann Oncol 2018, 29(3):535-537. https://doi.org/10.1093/annonc/mdy017

21. Kodahl AR, Ehmsen S, Pallisgaard N, Jylling AMB, Jensen JD, Laenkholm AV, Knoop AS, Ditzel HJ: Correlation between circulating cell-free PIK3CA tumor DNA levels and treatment response in patients with PIK3CA-mutated metastatic breast cancer. Mol Oncol 2018, 12(6):925-935. https://doi.org/10.1002/1878-0261.12305

22. Lee JH, Jeong H, Choi JW, Oh HE, Kim YS: Liquid biopsy prediction of axillary lymph node metastasis, cancer recurrence, and patient survival in breast cancer: A meta-analysis. Medicine (Baltimore) 2018, 97(42):e12862. https://doi.org/10.1097/MD.0000000000012862

23. Shah SP, Roth A, Goya R, Oloumi A, Ha G, Zhao Y, Turashvili G, Ding J, Tse K, Haffari G et al: The clonal and mutational evolution spectrum of primary triple-negative breast cancers. Nature 2012, 486(7403):395-399. https://doi.org/10.1038/nature10933

24. Kawazu M, Kojima S, Ueno T, Totoki Y, Nakamura H, Kunita A, Qu W, Yoshimura J, Soda M, Yasuda T et al: Integrative analysis of genomic alterations in triple-negative breast cancer in association with homologous recombination deficiency. PLoS Genet 2017, 13(6):e1006853. https://doi.org/10.1371/journal.pgen.1006853

\section{Figures}




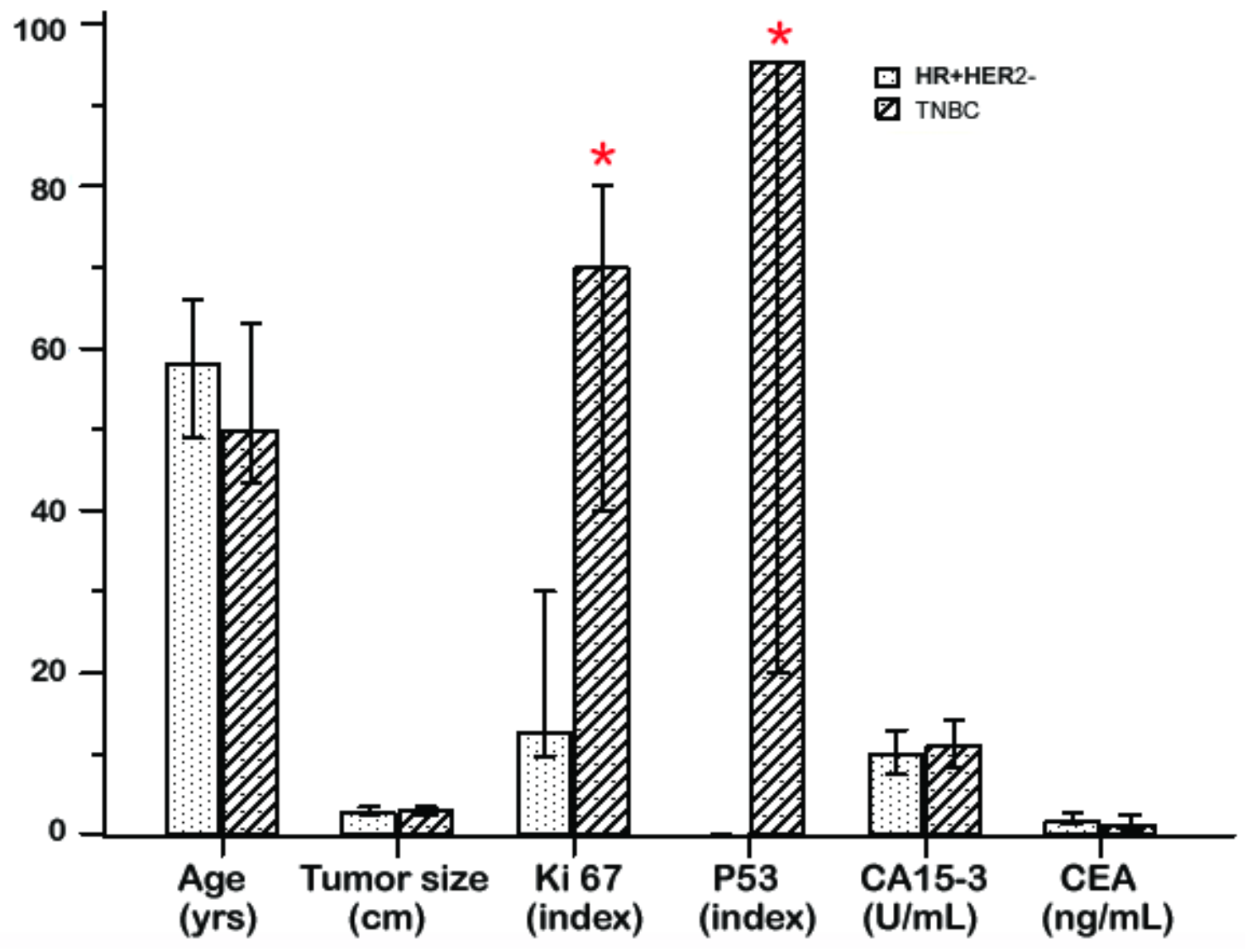

Figure 1

Comparison of the demographic and clinicopathological parameters between TNBC and HR+/HER2breast cancer patients. Columns and error bars represent medians and $95 \%$ confidence intervals, respectively. *, $p<0.05$; CA15-3, cancer antigen 15-3; CEA, carcinoembryonic antigen 


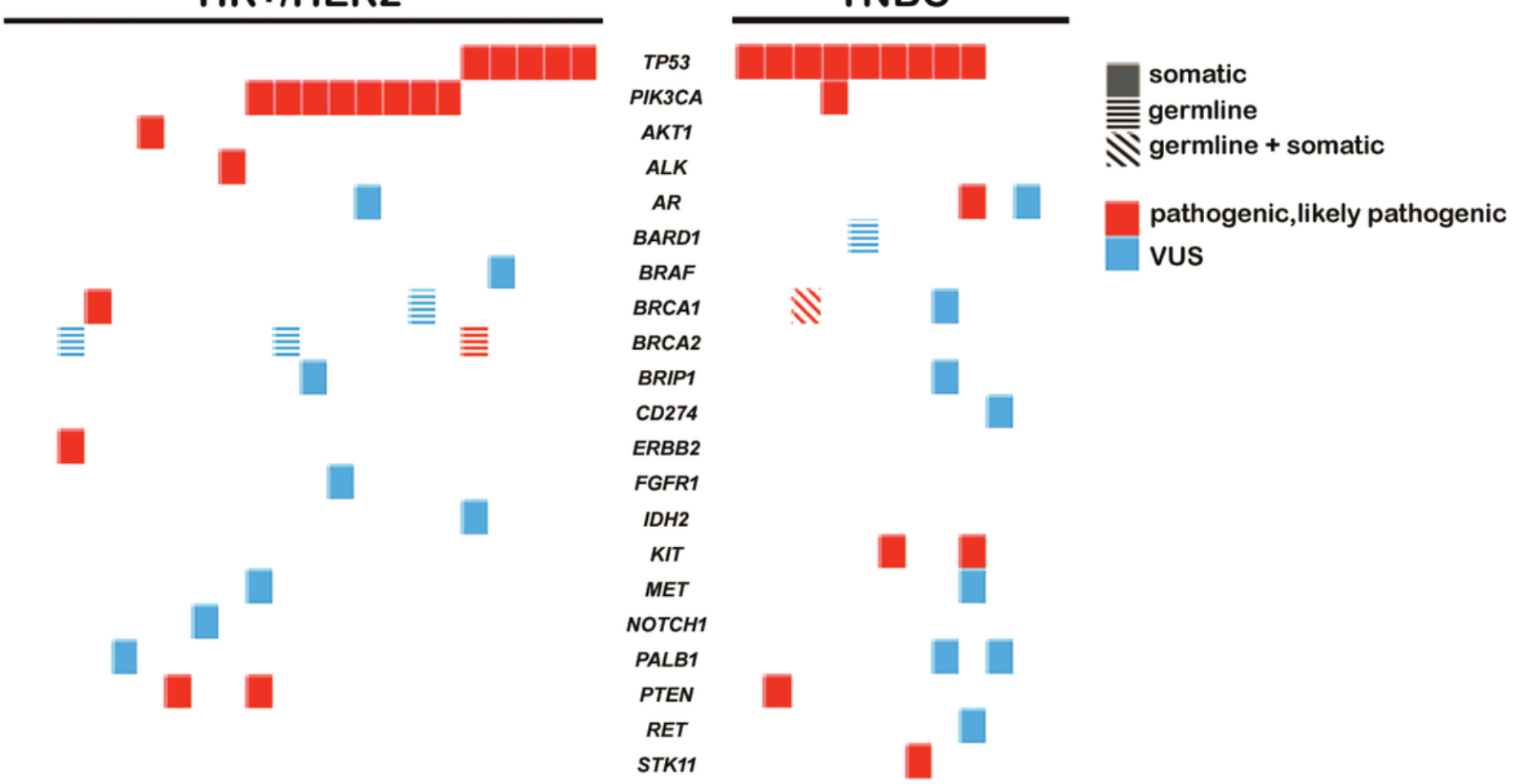

Figure 2

Mutation profiles of the two types of breast cancer. Each row represents a gene and each column represents a subject in the two groups of breast cancer patients.

a

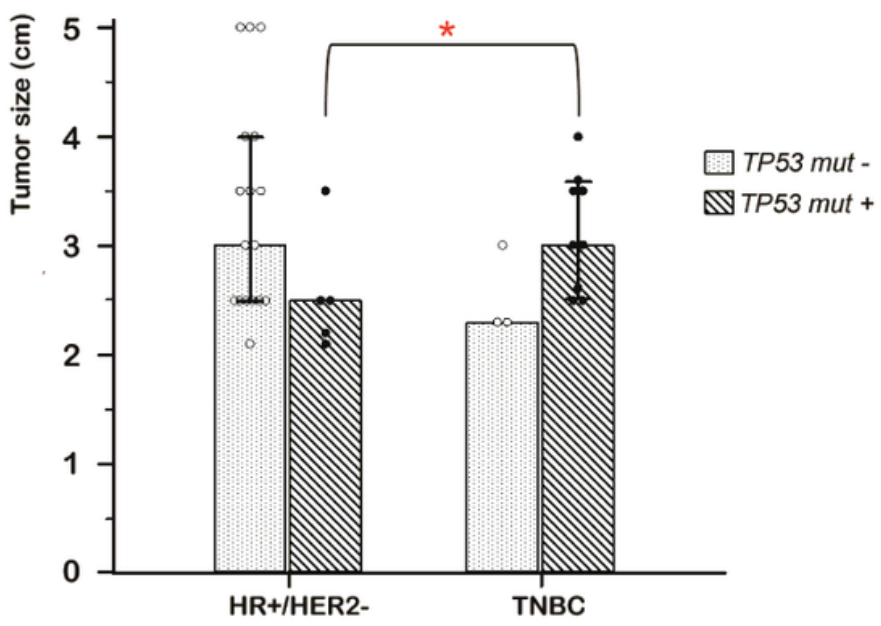

b

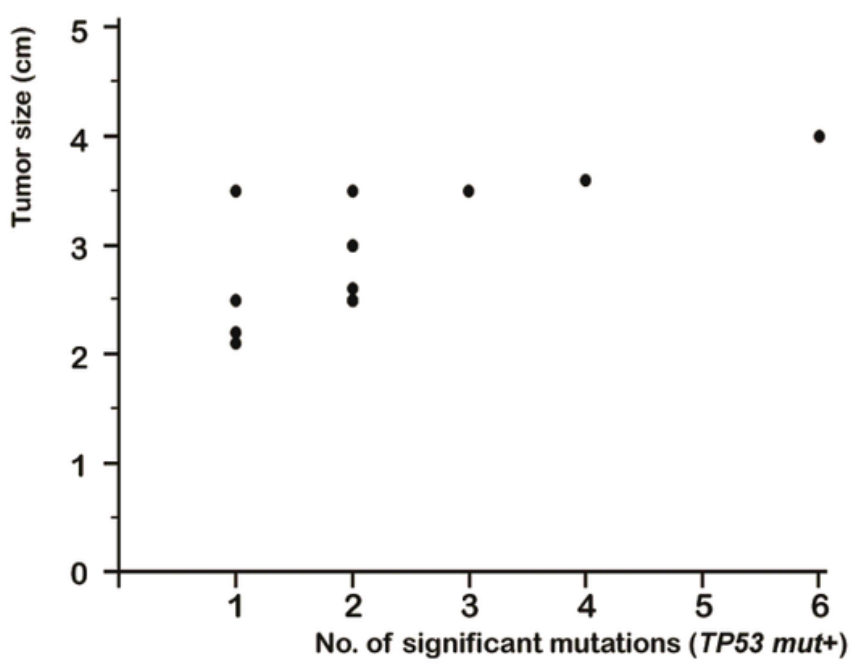

Figure 3

(a) Tumor size according to TP53 mutations in HR+/HER2- breast cancer and TNBC. In the HR+/HER2group, TP53-mutated cancers were smaller, whereas TP53-mutated tumors were relatively larger in the TNBC group. TP53-mutated TNBC was larger than TP53-mutated HR+/HER2- breast cancers $(p=0.0493)$.

(b) Tumor size according to the number of significant mutations in TP53-mutated breast cancer. 


\section{Supplementary Files}

This is a list of supplementary files associated with this preprint. Click to download.

- TableS1.docx

- Tables1.docx 